\title{
Diseño de Sistemas Tutores Inteligentes con Tecnología de Agentes: Los Agentes Docentes en el Módulo Tutor
}

\author{
Zulma Cataldi, Patricia Calvo, Fernando A. Salgueiro, Fernando J. Lage \\ liema@fi.uba.ar, pcalvo@fi.uba.ar, fsalgueiro@fi.uba.ar, flage@fi.uba.ar \\ LIEMA. Laboratorio de Informática Educativa y Medios Audiovisuales. \\ Facultad de Ingeniería. Universidad de Bs. As. \\ Universidad Tecnológica Nacional Facultad Regional Buenos Aires
}

\begin{abstract}
Resumen
A partir de un esquema básico, de interacción del alumno con los docentes, se estudian las diferentes características de los agentes para llevar a cabo una sesión de aprendizajes de tipo "uno a uno". Se propone un modelo de agente docente en el módulo tutor, y se reconoce a la herramienta a utilizar para el diseño de este sistema en el campo de los agentes, a fin de continuar con la siguiente etapa en la investigación.
\end{abstract}

Palabras-clave: Sistemas tutores inteligentes, sistemas multiagentes, módulo tutor

\begin{abstract}
A basic scheme, for interaction of students with professors as far as the agent characteritics from the agents in a session "one to one", is presented. A model of educational agent in the tutorial module sets out, and it is recognized the tool to use for the design of this system in the field of the agents, in order to continue the research.
\end{abstract}

Key-words: Intelligent tutoring systems, multiagent system, tutor module.

\section{$1 \quad$ Introducción}

Desde 2004, en el Laboratorio de Informática Educativa y Medios Audiovisuales. Facultad de Ingeniería. Universidad de Buenos Aires, se han venido realizando investigaciones tendientes a mejorar la enseñanza de la ingeniería y en particular de la Informática como una línea de trabajo propia, que por otra parte, se consolida a través de desarrollos informáticos que han sido transferidos a la comunidad educativa. En este contexto surge el proyecto en el cual se inscribe la presente comunicación, como una línea de trabajo que busca consolidar los esfuerzos tendientes a la mejora de la educación superior desde el ingreso del estudiante a la universidad como así también durante el tiempo que demande su carrera universitaria, que se centra en la enseñanza de la programación, que puede extenderse a otros dominios.

La preocupación se centra en el alto número de estudiantes por cada docente en los cursos iniciales de Programación Básica y en la búsqueda de opciones alternativas para facilitar los aprendizajes a fin de que el estudiante pueda obtener la retroalimentación necesaria para superar los requerimientos para permanecer en le nuevo ámbito educativo. En tal sentido, se han estudiado diferente tipos de aproximaciones a la solución, siendo una de ellas, la que se presenta a continuación a partir del paradigma de agentes inteligentes. ${ }^{1}$ Russell y Norvig [18] señalan que: un agente es un sistema capaz de percibir a través de sensores la informaciones que proviene del ambiente donde está insertado y reaccionar a través de efectores, por lo que se lo puede definir como una entidad de software que exhibe un comportamiento autónomo, situado en un ambiente en el cual es capaz de realizar acciones para alcanzar sus propios objetivos y a partir del cual percibe los cambios. Wooldridge y Jennings [24] expresan que: "Un agente es un sistema computacional que está situado en un ambiente y que es capaz de acciones autónomas en este medio para alcanzar sus objetivos de diseño”.

Un agente es inteligente cuando es capaz de actuar con autonomía y flexibilidad, basadas en cualidades de reactividad: o capacidad para percibir su entorno y responder a tiempo a los cambios que ocurren en él, proactividad o capacidad de mostrar un comportamiento dirigido por objetivos, tomando la iniciativa para planificar su actuación a fin de lograr sus objetivos y habilidad social desde la capacidad para interactuar por decisión propia en los procesos de negociación o cooperación con otros agentes de software o personas. Existen otras cualidades que

\footnotetext{
${ }^{1}$ Los resultados parciales de esta comunicación han sido presentados en la Revista Edutec [5], en el Workshop de Investigadores en Ciencias de la Computación WICC 2006 realizado en la Universidad de Morón [4] y en el Congreso Argentino de Ciencias de la Computación CACIC 2006 realizado en la Universidad de San Luis.
} 
incrementan la inteligencia del agente tales como: las actitudes mentales y el aprendizaje, y se suponen

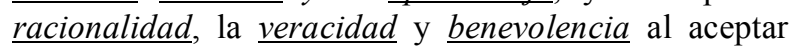
las solicitudes de servicio, siempre que sea posible.

En esta investigación, se busca una metodología que propicie el diseño de los STI con la integración de entidades o agentes inteligentes desde la visión que la inteligencia genuina sólo es posible si se cuenta con un cuerpo situado dentro de un entorno (modelo biológico), donde para interactuar con el medio ambiente, el agente debe ser capaz de percibir, razonar $y$ actuar, es decir debe poseer sensores que le permitan recolectar información, a fin de convertir esa información en conocimiento para alcanzar su objetivo razonando y actuando para modificar el propio entorno. En este enfoque inspirado en la sociología, se hace referencia también a conceptos como "comunicación", “cooperación”, “coordinación” y "competencia”.

Se trata pues, de obtener las bases teóricas conceptuales y metodológicas para la construcción de los STI donde la importancia de un tutor inteligente radica en que la captura de la experticia de los especialistas (uno o varios docentes expertos en el dominio), podrá ayudar a la formación de los estudiantes novatos, a través de la adecuación de la estrategia didáctica que mejor responda a las características de cada un de ellos.

Por este motivo, se pensó que un grupo de agentes cooperando a través del enfoque sociológico, podría aportar una buena opción, pero, este intento se debe tomar con ciudado, ya que en dominios complejos los sistemas multiagentes necesitan grandes cantidades de información acerca del mismo, por lo que resulta muy difícil prever todas las situaciones posibles para que los agentes puedan evolucionar y adaptarse al entorno.

Se propone diseñar las componentes de un STI con un marco teórico pedagógico basado en la Teoría Uno [16], es decir, mediante la integración de los conceptos planteados por la Teoría Uno al diseño computacional $[4,6]$.

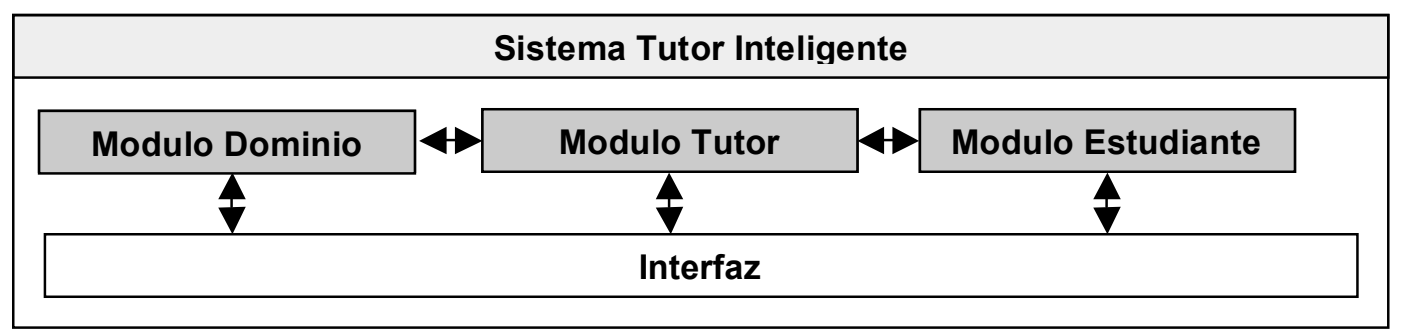

Figura 1: Arquitectura básica de Carbonell [5].

Siguiendo la idea de Carbonell [5], los STI, responden a una arquitectura trimodular sin solapamientos de funcionalidades y con interfaces bien definidas, a fin de obtener módulos independientes del dominio e intercambiables (ver Figura 1).

En comunicaciones previas, se han definido los requerimientos básicos de los Módulos: Tutor [19,20], Estudiante [3,8,9] y el Módulo Dominio, que ya sido estudiado en numerosas publicaciones [1, 15].

El Modulo Tutor del STI define y aplica una estrategia pedagógica de enseñanza, contiene los objetivos a ser alcanzados y los planes utilizados para alcanzarlos. Selecciona los problemas, monitorea el desempeño, provee asistencia y selecciona el material de aprendizaje para el estudiante. Integra el conocimiento acerca del método de enseñanza, las técnicas didácticas y del dominio a ser enseñado (ver Figura 2).

Consta de:

- Protocolos Pedagógicos: almacenados en una base de datos, con un gestor para la misma.

- Planificador de Lección: que organiza los contenidos de la misma.
- Analizador de Perfil: analiza las características del alumno, seleccionando la estrategia pedagógica más conveniente.

El Módulo Estudiante del STI tiene por objetivo realizar el diagnóstico cognitivo del alumno, y el modelado del mismo para una adecuada retroalimentación del sistema.

Se han planteado para el Módulo estudiante los siguientes submódulos (ambos almacenan los datos en una Base de Datos del Estudiante, con un gestor para la misma) (ver Figura 2).

- Estilos de aprendizaje: compuesto por una base de datos con los estilos de aprendizajes disponibles en el sistema, los métodos de selección de estilos y las características de cada uno de ellos [21,23].

- Estado de conocimientos: contiene el mapa de conocimientos obtenido inicialmente a partir del módulo del dominio y que progresivamente el actualizador de conocimientos irá modificando a través de los resultados obtenidos en las evaluaciones efectuadas por el módulo del tutor quien le enviará dichos resultados procesados. 


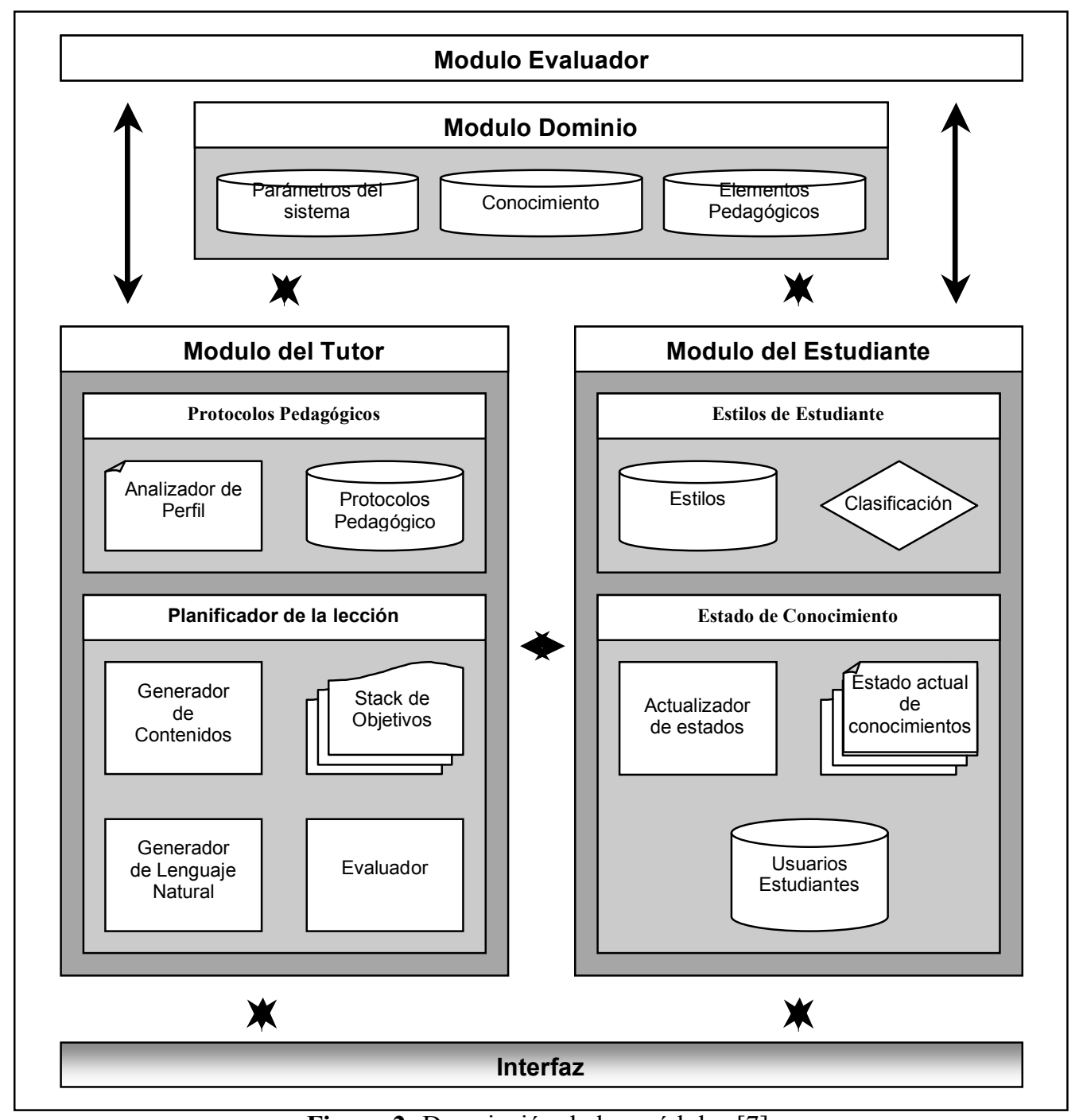

Figura 2: Descripción de los módulos [7].

El Módulo Dominio tiene el objetivo global de almacenar todos los conocimientos dependientes e independientes del campo de aplicación del Sistema Tutor Inteligente (STI) (ver Figura 2).

Entre sus submódulos están los siguientes:

- Parámetros Básicos del Sistema: los cuales se almacenan en una base de datos.

- Conocimientos: son los contenidos que deben cargarse en el sistema, a través de los conceptos, las preguntas, los ejercicios, los problemas y las relaciones.

- Elementos Didácticos: Son las imágenes, videos, sonidos, es decir material multimedia que se requiere para facilitarle al alumno apropiarse de conocimiento en la sesión pedagógica.

Los temas relacionados con el almacenamiento de conocimiento han sido tratados ampliamente por $[10,13,18]$.

\section{Metodología}

\subsection{Ventajas del paradigma multiagentes}

La Inteligencia Artificial Distribuida (IAD) se define como: "aquella parte de la inteligencia artificial que se centra en comportamientos inteligentes colectivos que son producto de la cooperación de diversos agentes" [12].

La aplicación del paradigma multiagentes al STI, supone el rediseño de la arquitectura para lograr mejores resultados. Entre las ventajas de la concepción multiagente se pueden citar:

- Tolerancia a fallas dado que un sistema creado por agentes autónomos no se colapsará cuando uno o más de sus componentes falle.

- Facilidades en la corrección de errores y en la modificación del sistema para proveer mejoras, dada la estructura modular del mismo basada en una estrategia "Divide $y$ Vencerás".

- Fuerte especialización de cada módulo del sistema.

- Posibilidad de que el sistema aprenda de las sucesivas evaluaciones que se realizan.

- El sistema es extensible, es decir que se le pueden agregar nuevas funcionalidades sin 
comprometer el funcionamiento previo del sistema.

- Es posible integrar múltiples plataformas, dada la estandarización de los mensajes entre agentes.

Como ya se señaló estas ventajas deben considerarse con mucha cautela, sobre todo en dominios complejos donde los volúmenes de información requeridos acerca del mismo son muy grandes, por lo que resulta muy difícultoso efectuar las revisiones de todas las situaciones posibles para que los agentes puedan evolucionar y adaptarse al entorno.

\subsection{Una arquitectura propuesta para el STI}

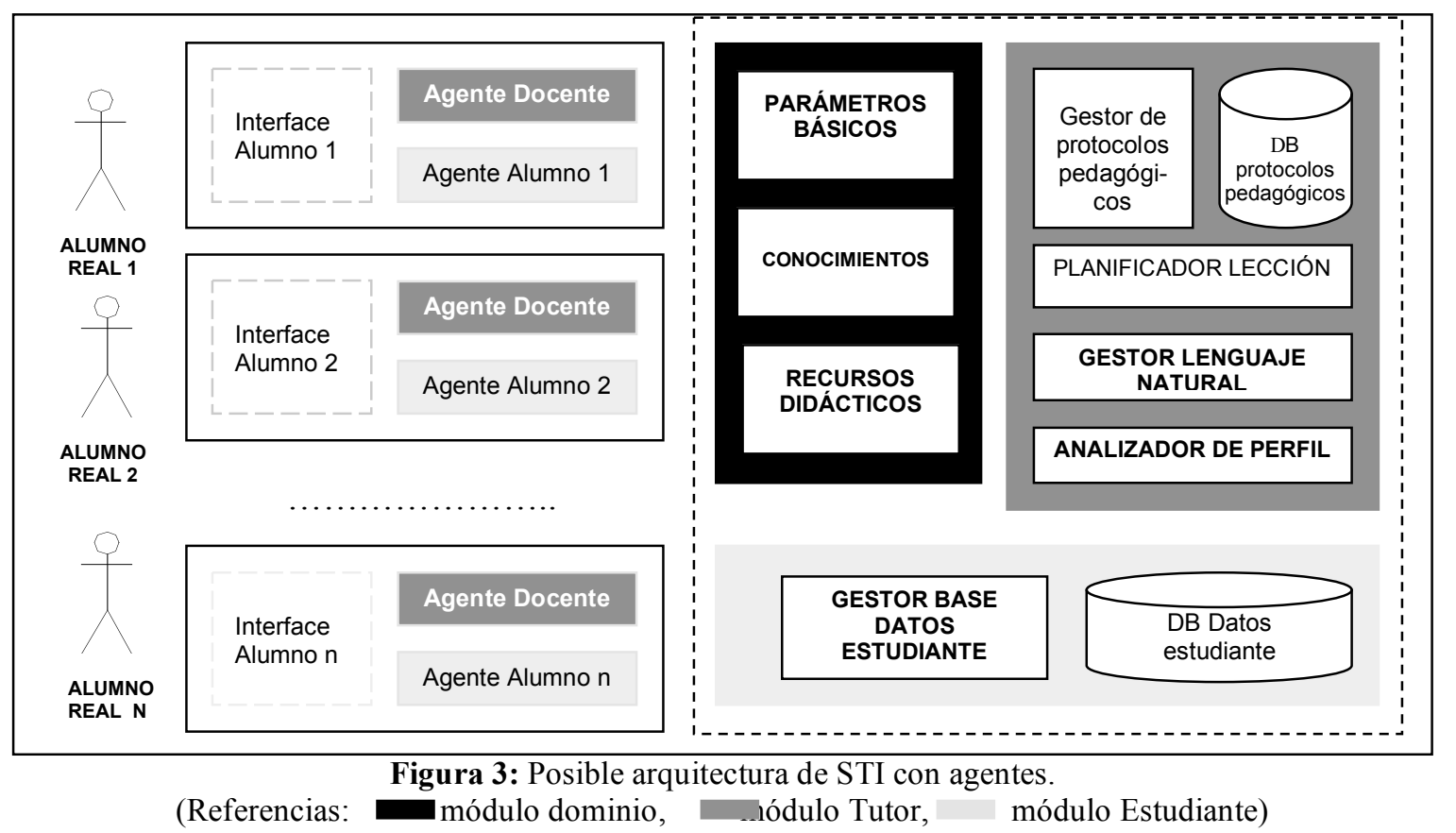

\subsection{Las características de los agentes del STI}

Un agente es reactivo si no incluye un modelo simbólico del mundo ni usa razonamiento simbólico complejo de ningún tipoy pueden construirse usando sistemas de producción, como propone Nilsson $[13,14]$ es decir definiendo conjuntos de reglas de producción y de producciones que indiquen la acción a realizar cuando se verifican determinadas condiciones. No existe comunicación explícita entre ellos, reaccionan a los cambios en el entorno generados por los otros agentes, a medida que los perciben.

Los agentes son deliberativos sí usan un modelo del mundo, explícitamente representado y funcionan siguiendo el paradigma de los sistemas clásicos de planificación en IA (Inteligencia Artificial), basados en el ciclo percepción-planificación-acción. Las intenciones se corresponden con la estrategia de acción que sigue el agente, y pueden ser reconsideradas.

Según Rao y Georgeff [17], un agente deliberativo lleva a cabo un ciclo infinito que sensa sus creencias, considera sus deseos para establecer cuáles se pueden alcanzar y los no alcanzables se eliminan, transformándose en intenciones, estableciendo planes de acción para la ejecución de las mismas, lo cual modificará nuevamente el entorno percibido por el agente, y su conjunto de deseos, dado que los alcanzados se eliminarán del conjunto.

Un agente es híbrido si dispone de componentes deliberativos para llevar a cabo razonamientos complejos, desarrollar planes y decidir, y simultáneamente tiene componentes reactivos para ten la reaccionar inmediatamente ante ciertos sucesos. Se adopta para este trabajo que: tanto los Agentes Docente como los Agentes Alumno son híbridos.

Por otro lado, los agentes que trabajan de forma independiente con sus propios objetivos constituyen sistemas independientes. $\mathrm{Si}$ tienen objetivos sin correlación son discretos, y en caso contrario son de cooperación emergente, cuando poseen mecanismos explícitos de cooperación. Esta categoría de SMA (sistema multiagente) puede considerarse como comunicativos cuando poseen y usan protocolos $\mathrm{y}$ procedimientos de cooperación, pero si usan medios indirectos, serán no comunicativos. El STI planteado es un sistema cooperativo y comunicativo.

Dadas las características del sistema, el diseño puede imponer para cada agente un protocolo de interacción y una estrategia, los cuales se construyen en función de los resultados sociales, dado que los agentes deben utilizar las estrategias impuestas. 
Para los planes consensuados, existen varios enfoques posibles. Para el caso del STI, se podría aplicar un enfoque de planeamiento multiagente, en el cual se establecen tres etapas, cada una llevada a cabo por uno o más agentes: elaboración de planes, coordinación y ejecución de los planes. Otro mecanismo posible es el de votación, en donde se somete a la decisión de los agentes las acciones posibles.

\subsection{Las tareas del módulo tutor}

Dentro del conjunto de tareas que el módulo Tutor debe considerar se encuentra las siguientes tareas:

- Mantener una jerarquía de metas que debe cumplir mientras traspasa los conocimientos al alumno quien cual producirá un resultado que el tutor no puede predecir de antemano. Para ello debe realizar las siguientes tareas:

- Identificar los objetivos para los alumnos (establece cuál es el objetivo de la sesión pedagógica que se está llevando a cabo y qué es lo que se pretende obtener como resultado para los estudiantes una vez finalizada dicha sesión). Para esto el agente docente usará la información sobre el alumno proveniente del agente alumno.

- Ser capaz de detectar las necesidades del alumno, y, en base a estos requerimientos, modificar la estrategia de enseñanza

- Supervisar y establecer el avance hacia los objetivos.

- Seleccionar los protocolos pedagógicos más eficientes para cada una de las sesiones pedagógicas a fin de impartirla de tal manera de lograr los mejores resultados con los elementos disponibles, que se pueden obtener a través del módulo del estudiante y del módulo del dominio.

Por otra parte, puntualmente en cada sesión debe:

- Presentar la explicación de un concepto (si es necesario de diferentes maneras, para variar el modo de presentar un tema si el alumno no alcanza a comprender el concepto nuevo). Esta tarea se realiza en colaboración con el agente Docente, el agente Alumno, el Gestor de Estrategias, el Módulo de Dominio y el Generador de Lenguaje Natural.

- Exponer numerosos ejemplos sobre los conceptos analizados utilizando al agente docente y al generador de lenguaje natural.

- Recibir las respuestas del agente alumno a las evaluaciones y las eventuales consultas del alumno.

- Ordenar y actualizar el registro de los nuevos conceptos adquiridos por los alumnos. Para esto el Agente Docente trabaja con el Agente Alumno.
- Supervisar las tareas que el alumno realice en colaboración con otros alumnos, como parte de su aprendizaje colaborativo.

- Comunicarse con los otros agentes Docentes, por ejemplo, para acordar tareas a realizar por los alumnos.

Considerando la variedad de tareas a realizar se propone un esquema de capas para el agente Docente pensado como un agente híbrido como se muestra en la Figura 3. En el esquema de la Figura 4 se observa la arquitectura del agente Docente, el cual por ser híbrido se lo pensó en tres capas. Los comportamientos que deban llevarse a cabo de acuerdo con reglas situaciónacción sin involucrar metas y objetivos se resuelven a nivel reactivo y las conductas dirigidas por objetivos (racionales o proactivas) se resuelven en los niveles superiores. En el nivel proactivo se implementan las conductas que permiten la cooperación con los otros agentes y la formulación de planes.

\subsection{La comunicación y la coordinación entre agentes}

En este modelo, el alumno real en ocasiones tomará una clase para adquirir conocimientos que imparte el sistema Tutor por medio del agente Docente que le corresponde, y en otras ocasiones realizará una tarea en colaboración con otros alumnos reales. Entonces, deberá recurrir mediante su agente alumno a otros alumnos reales. En este caso, los agentes Alumno se comunican entre si, para requerir colaboración en la realización de tareas en grupo. Se supone que los agentes Alumno (y Docente) son benevolentes, por lo que estarán predispuestos a colaborar con los otros y resolver los conflictos que pudieran surgir.

En algunos casos, es posible que un agente alumno sepa a qué otros agentes alumno debe recurrir; en otros casos, eventualmente requerirá de la ayuda de un agente mediador o facilitador de búsqueda para poder localizar a quien pueda cooperar con él para llevar cabo la tarea asignada.

El uso de agente mediador para el intercambio de mensajes resuelve el problema de la eventual saturación de mensajes entre agentes Docentes y también entre agentes Alumno.

La comunicación por medio de mensajes permite implementar estrategias complejas de coordinación. Por lo general, se trata de un emisor y un receptor, pero existe también la posibilidad de utilizar el "broadcasting" como técnica para enviar un mensaje a un grupo de agentes, es decir un emisor y varios receptores. Otra forma posible es la pizarra que surge como un sistema de comunicación particularmente útil para el trabajo en colaboración. Se trata de una estructura de datos para la comunicación general gestionada por un controlador que se constituye en un área común de trabajo, permitiendo compartir la información, de modo tal que un grupo de agentes pueda "leer" y "escribir" en el área. 
Por otro lado, la interface con el alumno real puede recordar los hábitos y preferencias del usuario, $\mathrm{y}$ adaptarse a las características propias de cada alumno real, de modo de ofrecer una aproximación amigable al STI.

En cuanto a la evaluación del mecanismo de interacción y sociabilidad, en este sistema es posible realizar un análisis sobre el comportamiento emergente y la sociabilidad usando el test de Huhns y Singh [11], que permite medir las relaciones entre los agentes. La regla del test enuncia: "A system containing one or more reputed agents should change substantively if another of the reputed agents is added to the system".

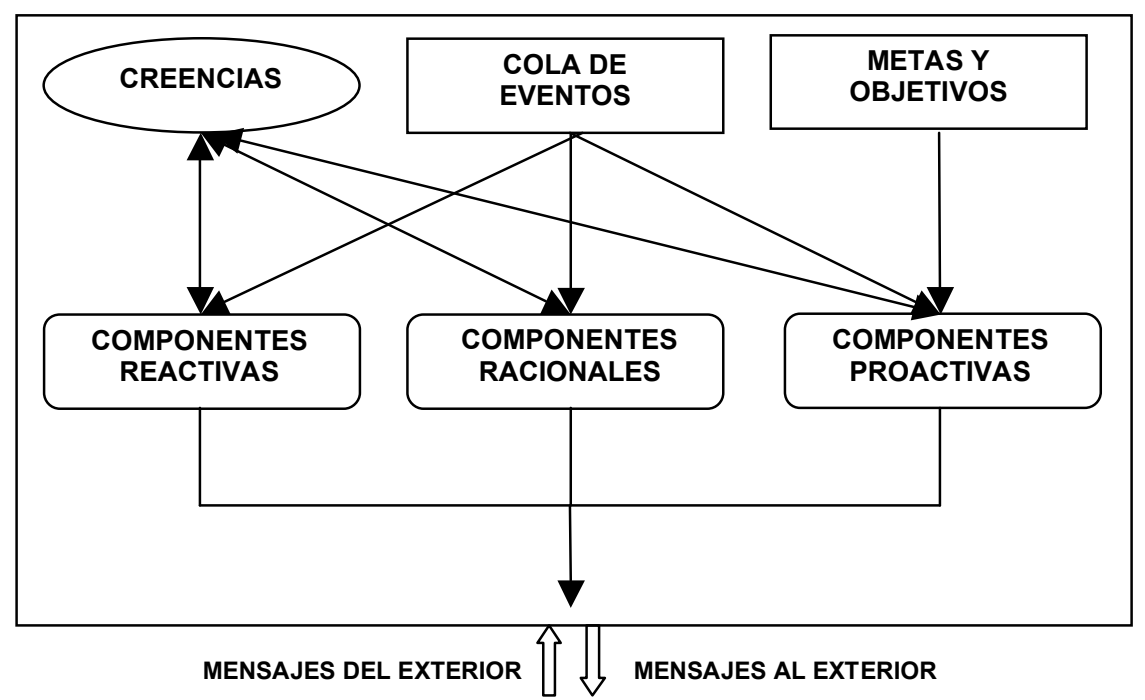

Figura 4: Esquema de tres capas del agente Docente

El cambio hace referencia al comportamiento de los agentes, siendo un cambio válido que proporciona una mejora en el rendimiento del sistema.

Huhns y Singh [11], proponen este test basado en la sociabilidad de los agentes que se manifiestan a través de cambios en su estructura. Es decir, cómo se puede especificar esta propiedad lo mejor posible para poder aplicar el test por cualquier desarrollador $\mathrm{o}$ analizador de agentes. Aunque es test no es simple matemáticamente, se lo puede aplicar a través de las ideas clave que los autores señalan y discuten. Las condiciones especificadas para el test son posibles de ser verificadas y éstas son: que el entorno del agente no sea estático, que sea suficientemente observable, y que los agentes sean del mismo tipo. Los componentes principales de la especificación son: el ambiente en el cual los agentes existen y la formulación de la sociabilidad, que combinados producen la formulación del test a aplicar. El criterio de evaluación del mecanismo de interacción establece el modo de medir tal interacción del sistema. Puede usarse como criterio el grado del beneficio global de los agentes (criterio de bienestar social), o bien determinar si un resultado es Pareto óptimo, es decir que no existe otro resultado en el que por lo menos un agente obtenga un beneficio mayor y ningún otro agente obtenga un beneficio menor en otro resultado.

\section{$3 \quad$ Resultados}

\subsection{Las actividades a realizar en las sesiones de aprendizaje}

A fin de delimitar las posibles acciones requeridas por los componentes del sistema cuando un alumno real se conecta al STI para tener una sesión, ésta puede tener como objetivo una de las dos actividades siguientes:

a) la adquisición de un concepto o habilidad nueva (sesión de aprendizaje "uno a uno" con su docente),

b) la realización de un trabajo en colaboración con otros alumnos reales, eventualmente pendiente de una sesión anterior (sesión de aprendizaje colaborativo).

En el primer caso, el desarrollo de la sesión se centrará en una interacción con retroalimentación entre el docente y su alumno, con posterior evaluación. El segundo caso se da cuando un agente docente propone al alumno real realizar un trabajo en colaboración con otros alumnos reales y éste responde afirmativamente, el agente alumno registrará la elección del alumno real y se comunicará con otros agentes alumnos para proponer realizar dicho trabajo en colaboración. Cuando un agente alumno recibe una propuesta de trabajo en colaboración, y ya se encuentra comprometido en algún trabajo inconcluso, rechazará la solicitud. Si no es así, primero solicitará "permiso" a su agente Docente, el cual, en base a los objetivos 
propuestos para ese alumno y el grado de avance en sus aprendizajes, otorgará o no el permiso. Si el agente Docente aprueba el trabajo, el agente Alumno consultará al alumno real para saber si desea realizar dicho trabajo y si el alumno real llega a un acuerdo, el agente Alumno registrará su elección.

\subsection{El módulo tutor $y$ el armado de la sesión de aprendizaje “uno a uno.}

Se debe llevar a cabo el análisis del Módulo Tutor comenzando con el caso de armado de una sesión de aprendizaje. Es decir, una vez registrado en el sistema y "logeado" el alumno, el agente Docente recibirá como entrada, las características del estilo de aprendizaje del alumno y el estado del conocimiento del mismo provenientes del Módulo Alumno. Se lleva a cabo el análisis de perfil del alumno, y se solicita al
Gestor de Protocolos Pedagógicos el que se corresponda a las características del alumno. Luego se le solicita al Planificador de la Lección que construya la misma utilizando los elementos del Módulo del Dominio, por lo que se genera la pila de objetivos de la lección y el procesador de lenguaje natural toma la información contenida en las estructuras internas y finalmente prepara la salida en lenguaje natural, que el Agente Docente presenta al alumno utilizando la interface.

A partir de la evaluación posterior y la comparación con los objetivos propuestos surgirán las modificaciones eventuales a la información del alumno, o a la selección de estrategias más convenientes para la continuación del proceso de aprendizaje, las cuales serán almacenadas en la base de datos del alumno, de acuerdo al ciclo de la Figura 5.

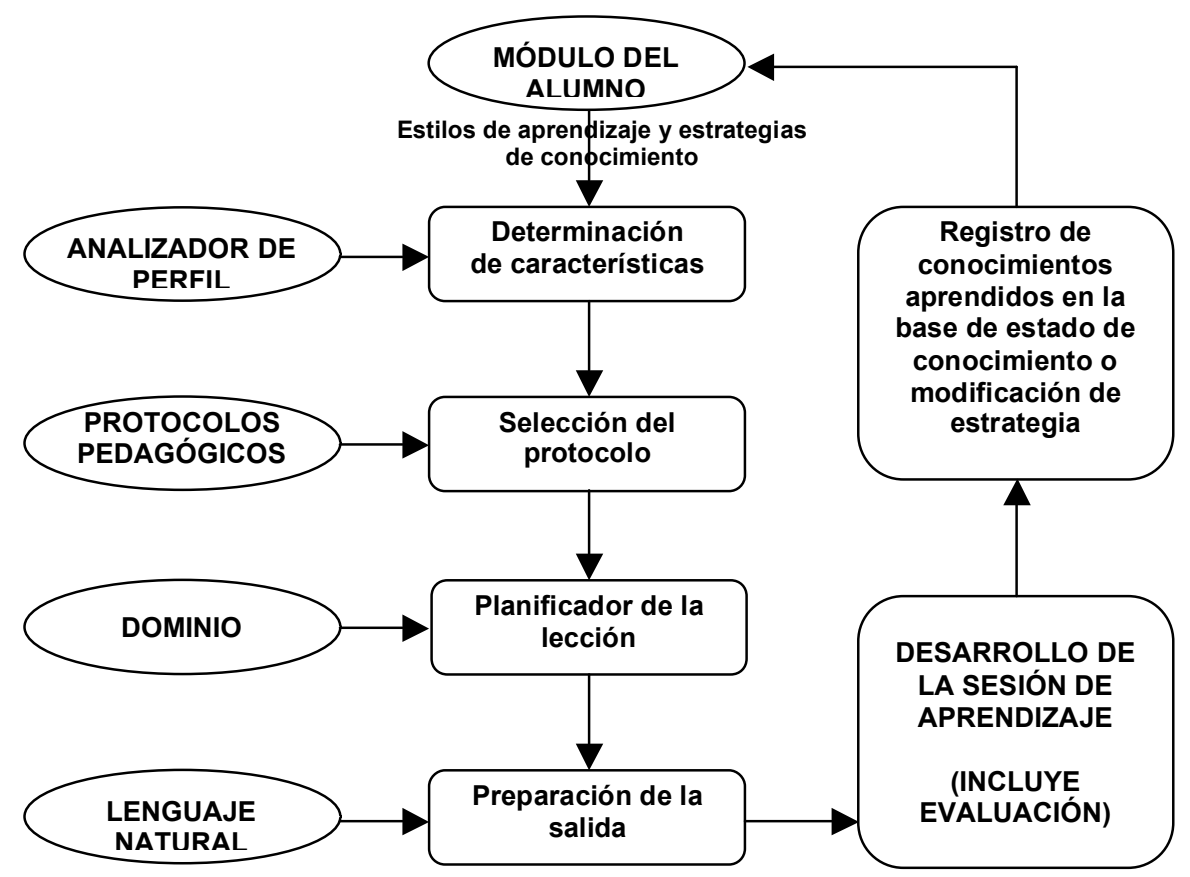

Figura 5: El agente Docente en una sesión "uno a uno."

En la Figura 5 se presentan los pasos a seguir por el agente Docente cuando se prepara y lleva a cabo una sesión "uno a uno" con un alumno. A partir de los datos recibidos del módulo Alumno y del análisis de su perfil, se selecciona el protocolo más adecuado, y se prepara la lección, tomando los contenidos del Módulo Dominio y con el auxilio del Procesador de Lenguaje Natural se prepara la salida. Una vez desarrollada la Sesión de Aprendizaje, se realizará una evaluación, a partir de la cual se registrarán los nuevos conocimientos del alumno si ésta ha sido superada; en caso contrario, se procederá a modificar la estrategia utilizada.

\section{Discusión}

\subsection{Consideraciones para el diseño de los agentes}

Para llevar a cabo este diseño, el AUML (Agent Unified Modelling Language) resulta una herramienta de gran utilidad, ya que toma herramientas del UML (Unified Modelling Language) y las utiliza en el campo de los agentes, es decir, extiende el UML permitiendo la especificación de protocolos de interacción de agentes y la representación de estructuras sociales y organizativas entre agentes.

Para esto, AUML agrega al UML los diagramas de protocolos, que son diagramas de secuencias UML para especificar una colección de mensajes, parcialmente ordenados, entre un agente emisor y un 
agente receptor. Bauer, Odell et al. [2] sugieren una representación en tres capas, denominada Agent Interaction Protocol (AIP), que representa los protocolos de comunicación que utiliza paquetes y los templates de UML para la especificación de estos protocolos.

\section{Conclusiones}

Se ha presentado un esquema del módulo tutor y cómo se gestiona el uso del sistema por los estudiantes en los casos en que la sesión se realiza para tutorizado "uno $a$ uno" o a través el diálogo entre pares en colaboración. Se han analizado las características que deben tener los agentes que participarán en el subsistema AlumnoDocente delimitando el tipo de interacciones a considerar.

Se ha investigado el problema integrando distintas perspectivas: los sistemas inteligentes y las ciencias de la educación, a fin de obtener una base teórica consolidada, sobre la cual desarrollar un tutor inteligente. Se han tenido en cuenta particularmente el análisis de: las teorías de aprendizaje, de enseñanza y las características de los sistemas multiagentes que pudieran aportar a la propuesta.

\subsection{Trabajos futuros}

La arquitectura presentada permite una forma efectiva de comunicación entre el usuario y el sistema, ya que considera el estilo de aprendizaje del estudiante como variable de entrada. Una vez determinado el marco teórico y los requerimientos de los módulos mutiagentes, se seleccionarán las herramientas metodológicas y de desarrollo más adecuadas a fin de: a) Llevar a cabo el diseño del módulo de agentes Docentes para la tarea de generación de estrategias de enseñanza en el módulo del tutor, que permita la adición de nuevos protocolos de enseñanza que se adapten a las necesidades de cada del alumno (a través de su perfiles) y b) Llevar a cabo el diseño de un agente (modelo de estudiante) que permita obtener los diferentes estilos de aprendizaje de los estudiantes de acuerdo sus preferencias.

Estas etapas permitirán arribar a la metodología más apropiada para trabajar con sistemas multiagentes en el diseño de los sistemas para tutorizado inteligente.

\section{$6 \quad$ Agradecimientos}

Este trabajo ha sido desarrollado en el marco de los Proyectos: Modelado del tutor basado en redes neuronales para un sistema tutor inteligente C099 y Metodología de diseño de Sistemas Tutores Inteligentes con Tecnología de Agentes. 2005-2006 de la Universidad Tecnológica Nacional, Facultad Regional Buenos Aires en colaboración con el Laboratorio de Informática Educativa y Medios Audiovisuales (LIEMA) de la Facultad de Ingeniería, de la Universidad de Buenos Aires.

\section{Referencias}

[1] ANDERSON, J.R.; CORBETT, A.T.; KOEDINGER, K.R.; PELlETIER, R. Cognitive tutors: Lessons learned. Journal of the Learning Sciences. 1995. 4(2), 167-207.

[2] BAUER, B. MULLER, J.P. AND ODELL, J. Agent UML: A Formalism for Specifying Multiagent Interaction. P. Ciancarini and M. Wooldridge, editors, Agent-Oriented Software Engineering, pages 91-103. Springer-Verlag, Berlin, 2001.

[3] BLOOM, B. S. The 2 sigma problem: The search for methods of group instruction as effective as one-to-one tutoring. Educational Researcher 1984, 13, 4-16.

[4] CALVO, P., CATALDI, Z; SAlguEIRO, F.; COSTA, G.; MÉNDEZ, P., RENDÓN ZANDER, J. Y LAGE, F. Sistemas Tutores Inteligentes basados en agentes. Anales del VIII Workshop de Investigadores en Ciencias de la Computación. Págs. 671-675. ISBN13: 978-950-9474-34-5, 1 y 2 de junio 2006.

[5] CARBONELL, J. R. AI in CAI: An artificial intelligence approach to computer assisted instruction. IEEE transaction on Man Machine System. V11 n.4, p 190-202. 1970.

[6] CATAldi, Z; SAlgueIRO, F. Y LAGE, F. Sistemas tutores multiagentes con modelado del estudiante y del tutor. Revista Edutec. ISSN: 11359250. Núm. 20. Enero 2006. Pág.1-22.

[7] CHI, M. T. H., SILER, S., JEONG, H., YAMAUCHI, T., Y HAUSMANN, R. Learning from human tutoring. Cognitive Science 2001.25, 471-533.

[8] COSTA, G.; SAlgueiro, F. A., CATALDI, Z., GARCÍA-MARTÍNEZ, R. Y LAGE, F. J. Sistemas inteligentes para el modelado del estudiante Proc. GCETE'2005 CD, Global Congress on Engineering and Technology Education.Brasil. marzo 13-15 2005.

[9] ENGSTRÖM, Y. Perspectives on activity theory. London; New York: Cambridge University Press, 1999.

[10] GENESERETH, M. AND NILSSON, N. Logical Foundations of Artificial Intelligence. San Francisco: Morgan Kaufmann 1987.

[11] HUHNS, M. y SINGH, M.P. A Multiagent Treatment of Agenthood Applied Artificial Intelligence, Volume 13, Numbers 1-2, 1 January 1999.

[12] IGLESIAS, C.A., GARIJO, M., GONZÁLEZ, J.C. Metodologías orientadas a agentes Revista Iberoamericana de Inteligencia Artificial, Numero 6, Volumen 2, Otoño 1998.

[13] NILSSON, N. (1998) Artificial Intelligence: A New Synthesis. Morgan Kaufmann Publishers.

[14] NILSSON, N.J. Logic and Artificial Intelligence. Artificial Intelligence 1991. 47: 31-56.

[15] PAGÉS, C., MARTINEZ, J.; GUTIERREZ, O., DIEZ T. Sistema Inteligente de Tutorización 
Avanzada (SITA). Un caso de aplicación: GEKA. Publicado en RED. Revista de Educación a Distancia Publicación en línea. Murcia (España). Año IV. Número monográfico II. 2005.

[16] PERKINS, D. La escuela inteligente. Gedisa. 1995.

[17] RAO, A. S., y GEORGEFF, M. P. BDI Agents: From Theory to Practice, In Proccedings of the First International Conference on Multi-Agent Systems (ICMAS 1995), São Francisco, MIT Press, pp. 312-319.

[18] RUSSELL, S. J. y NORVIG P. Artificial Intelligence: A Modern Approach (2nd Edition). Prentice Hall. 2003.

[19] SALGUEIRO, F. A. Tesis de Grado en Ingeniería Informática: Sistemas inteligentes para modelado del tutor. Facultad de Ingeniería. Universidad de Buenos Aires. 2005.
[20] SAlGUEIRO, F. A., COSTA, G., CATALDI, Z., GARCÍA-MARTÍNEZ, R. Y LAGE, F. J. Sistemas inteligentes para el modelado del tutor. Proc. GCETE'2005 CD, Global Congress on Engineering and Technology Education.Brasil. marzo 13-15. 2005.

[21] SLEEMAN, D. H., y BROWN, J. S. Intelligent tutoring systems. New York: Academic Press. 1982.

[22] VYGOTZKY, L. Mind in society. The Development of Higher Psychological Process. Cambridge. N. A. Harvard University Press. 1978.

[23] WENGER, E. Comunidades de práctica. Aprendizaje, significado e identidad. Barcelona: Paidós, 2001.

[24] WOOLDRIDGE, M. J. y N. R. JENNINGS Agent Theories, Architectures and Languages: A survey. In M. J. Wooldridge and N. R. Jennings (Eds.), Intelligent Agents, Vol. 890 of LNAI. 1995. 